\title{
Comparison of Preparation Methods for the Evaluation of Nerve Axons
}

\author{
By \\ Yuji ISHIDA $^{\text {a) }}$, Noboru GOTO ${ }^{\text {a,b) }}$, Kaori CHIBA ${ }^{\text {a) }}$, Yuuki SOUMA ${ }^{\text {a) }}$ and \\ Jun GOTO ${ }^{\text {b) }}$
}

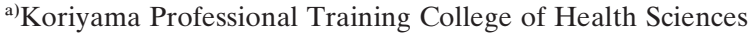

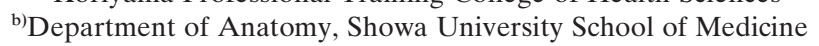

\begin{abstract}
Summary: We have conducted a study to compare various preparation methods, including a certain number of new methods, to find which ones are best suited to the morphological evaluation of nerve fibers in the pyramidal tract of the human medulla oblongata. Our main concern was to find fixation and staining methods that would minimize errors, especially regarding the tissue shrinkage ratio and the ease of staining. From the two fixation methods we examined, the most satisfactory was the secondary chromic acid fixation (which gave the best overall results when followed by nitrocellulose embedding), as it gives the lowest shrinkage ratio with the narrowest range $(10 \pm 0 \%)$. Among the ten staining methods we tested, we found that the most suitable for morphological evaluation were the discriminative staining methods (Luxol fast blue-Periodic acid-Schiff-Hematoxylin stain, Masson-Goldner-Goto method and modified Hematoxylin-Eosin stain) and the silver impregnation methods (Luxol fast blue-Silver impregnation and Luxol fast blue-Silver impregnation-Periodic acid-Schiff-Hematoxylin).
\end{abstract}

- Received for Publication, January, 16, 2008 -

Key Words: Axon, Discriminative staining method, LPH, Nerve fiber, Nervous pathway

The nervous pathways consist of chains of neurons consisting of neuronal somata and nerve fibers. Among the various nerve descending pathways that exist in the human nervous system, one of the most important is the pyramidal tract, which controls the movements of skeletal muscles. (Descending pathways also include the rubrospinal, tectospinal, vestibulospinal, reticulospinal, interstitiospinal and solitariospinal tracts, as well as hypothalamospinal fibers, propriospinal pathways, etc.)

In the pyramidal tract, there are two kinds of neuronal chains: (a) the corticospinal tract, and (b) the corticonuclear tract. Past studies up to now have inclined toward considering the neuronal somata as being the origin of various pathways, so that there have been no proper morphometric evaluation of nerve fibers. One reason for this lack of data could be the limitations of the various methods, including silver impregnation methods, used to evaluate nerve fibers, especially nerve axons.
We have conducted this study to compare various preparation methods, including new methods, to find which ones are the most suitable for the morphological evaluation of nerve fibers in the corticospinal tract.

\section{Methods}

We used several human medullae oblongatae from anatomy dissection as material for this study. None of the various histological preparation methods used for the study of nervous tissue, is able to evaluate satisfactorily both axons and surrounding myelin sheaths. Silver impregnation methods used to be the only reliable methods for axons.

We compared several preparation methods by examining several factors: fixation, embedding, sectioning, staining and tissue shrinkage. We selected the following staining methods to evaluate the findings concerning the nerve fibers: Luxol fast

Correspondence: N. Goto, MD, Koriyama Professional Training College of Health Sciences, 9-3, Zukei 2, Koriyama, Fukushima $963-$ 8834, Japan. Telephone: 024-936-7777, Facsimile: 024-936-7788, e-mail: n-goto@k-tohto.ac.jp, goto@sea.plala.or.jp (Dr. Goto’s private e-mail) 


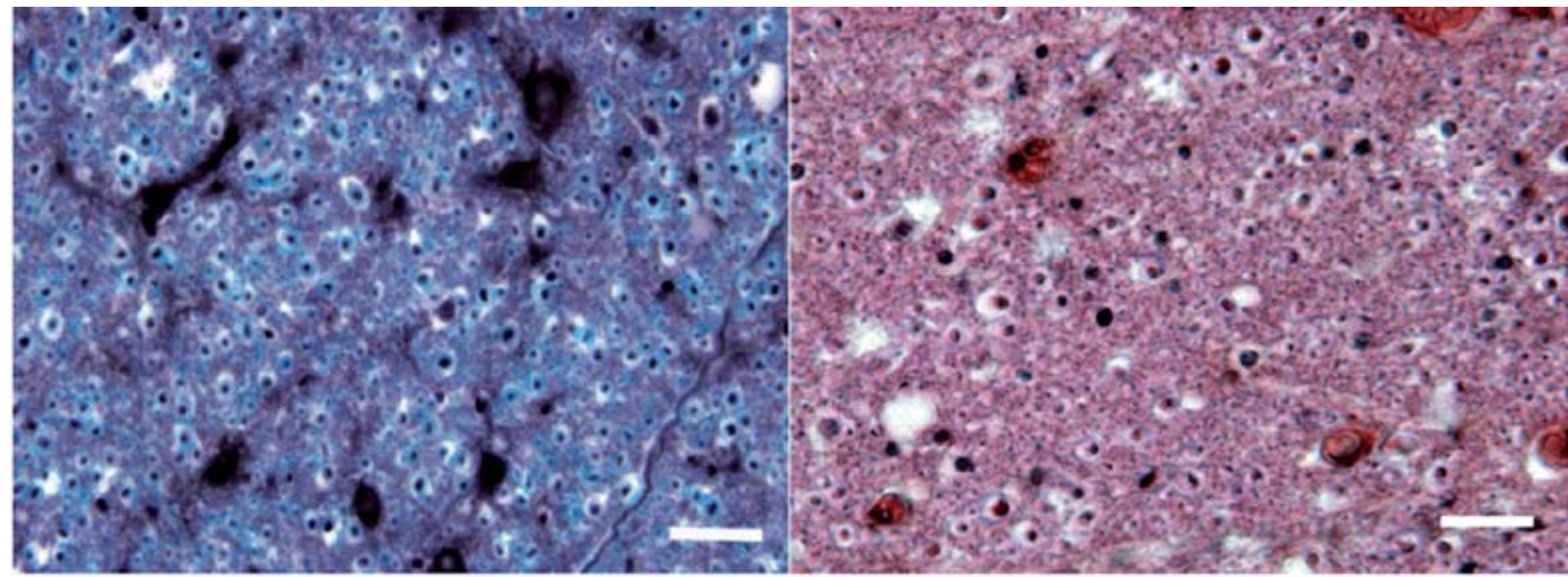

Figure 1

Figure 2
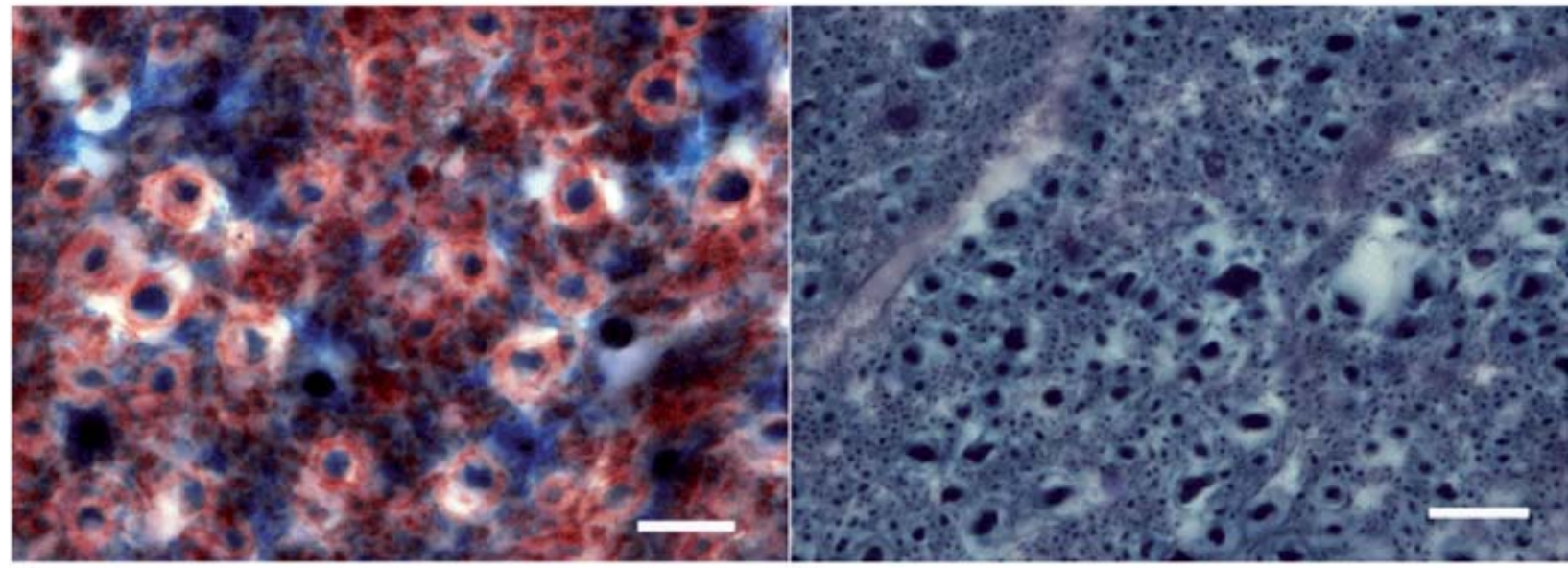

Figure 3

Figure 4

Fig. 1. High power view of the human medullary pyramid (LPH)

Luxol fast blue-Periodic acid Schiff-Hematoxylin triple stain based on secondary chromic acid fixation and nitrocellulose embedding. Bar $=10 \mu \mathrm{m}$.

Fig. 2. High power view of the human medullary pyramid (modified HE)

Modified Hhematoxylin-Eosin: Conventional HE stain yields axonal stains after secondary chromic acid fixation and nitrocellulose embedding. Bar $=10 \mu \mathrm{m}$.

Fig. 3. High power view of the human medullary pyramid (MGG)

Trichrome staining method of Masson and Goldner modified by Goto ${ }^{3)}$. The MGG method can only distinguish astrocytic fibers from unmyelinated axons. Bar $=10 \mu \mathrm{m}$.

Fig. 4. High power view of the human medullary pyramid (LS)

Luxol fast blue-Silver impregnation method is highly recommended for the morphometry of axons, especially after secondary chromic acid fixation and nitrocellulose embedding. However, ordinary Silver impregnation methods with conventional fixation are not suitable due to the severe shrinkage of axons. Bar $=10 \mu \mathrm{m}$.

blue-Periodic acid Schiff-Hematoxylin $(\mathrm{LPH})^{1)}$, Luxol fast blue-Silver impregnation-Periodic acid Schiff-Hematoxylin (LSPH) ${ }^{1}$, , Luxol fast blue-Silver impregnation method (LS) ${ }^{1)}$, Masson-GoldnerGoto $(\mathrm{MGG})^{1)}$ method, modified Hematoxylin-
Eosin $(\mathrm{mHE})^{1)}$ stain, conventional HematoxylinEosin (HE) stain, Mallory's Phosphotungustic acidHematoxylin (PTAH) stain, Kultschitzky's myelin sheath stain (KMS), Luxol fast blue-Cresyl violet stain of Klüver and Barrera (K-B), Silver 
impregnation methods of Bielschowsky (SIB) type and Hirano's silver imprengnation for Celloidin $(\mathrm{HSI})^{2)}$, etc.

\section{Results}

Fixation: We compared the conventional fixation method, with $3.6 \%$ formaldehyde, and the combined method, with secondary chromic acid fixation following formaldehyde fixation. Regardless of the embedding methods used, LPH and HSI resulted in good axonal stains after the formaldehyde + secondary chromic acid fixation, while with only the formaldehyde fixation, no stains appeared, except with silver impregnation methods.

Embedding: Secondary chromic fixation caused $10 \pm 0 \%$ (in length) shrinkage of nitrocellulose sections. However, conventional paraffin embedding without secondary chromic acid fixation yielded a wide range of shrinkage $(30-70 \%$ in length). Nitrocellulose embedding after formaldehyde fixation without secondary chromic acid fixation also yielded around $40 \%$ shrinkage in length.

Sectioning: Transverse sections were prepared at 20 or $30 \mu \mathrm{m}$ thickness for the nitrocellulose blocks, and at $10 \mu \mathrm{m}$ thickness for the paraffin blocks in order to get a transverse view of nerve fibers. The sectioning itself was easier with nitrocellulose blocks, than with paraffin blocks.

Staining: Among staining methods described above, LPH, LSPH, MGG methods and mHE yielded satisfactory results in axonal stains. These are shown in Figs. 1-4, together with brief explanations. Conventional staining methods (HE, PTAH), however, showed poor results. KMS and K-B methods showed no stains of axons. While they are of great value with secondary chromic acid fixation for morphometry of axons, SIB and HSI for Celloidin methods, usually without secondary chromic acid fixation, showed poor results, with severe shrinking of axons.

\section{Discussion}

Our thorough evaluations revealed that the most suitable preparation methods for the staining of nerve fibers in the nervous pathways are the following, in descending order: (1) LPH, (2) mHE, (3) MGG method and (4) LS. These evaluations were conducted taking into account two different view points: First, it is of the utmost importance to mark the axons properly. Discriminative staining methods $\left(\right.$ Goto, 1987) ${ }^{1)}$ represented by LPH are the most suitable methods for this purposes. Besides LPH, these staining methods also include LSPH and LS. However, Bielshowsky-type silver impregnation methods are far less suitable, as they produce highly shrinked axons when used with only formaldehyde fixation. It is therefore necessary to employ secondary chromic acid fixation and nitrocellulose embedding. Electron microscopy can be applied only to a very small area. PTAH is a good staining method for glial fibers (especially astrocytic fibers), but it does not work well on axons. Although K-B stain and myelin sheath stains using hematoxylin (Weigert type) such as Kultschitzky method can stain myelin sheaths nicely, they usually do not work on axons. Obviously, some of the methods described above, such as conventional HE, PTAH, KMS, K-B, are not suitable for evaluations of axon sizes.

Secondly, it is most important to obtain simultaneous staining of both axons and myelin sheaths, as with $\mathrm{LPH}$ and MGG methods for the purpose of dividing nerve fibers into myelinated and unmyelinated. The LPH method stains axons in black, for both myelinated and unmyelinated fibers, whereas myelin sheaths appear colored in blue. The MGG method can only distinguish astrocytic fibers from unmyelinated axons.

\section{References}

1) Goto N: Discriminative staining methods for the nervous system: Luxol fast blue-Periodic acid-Schiff-Hematoxylin triple stain and subsidiary staining methods. Stain Technol 62:305-315, 1987.

2) Hirano A, Zimmerman HM: Silver impregnation of nerve cells and fibers in celloidin sections. Arch Neurol 6:114-122, 1963.

3) Goto J, Goto N, Ezure H, Nonaka N, Ma X: Morphological differentiation of nerve fibers: central, peripheral, myelinated and unmyelinated. Okajimas Folia Anat Jpn 77:211216, 2001. 
